\title{
基于缺电子烯胺交叉氨化过程的多组分反应研究进展
}

\author{
曹 硕 $a$ 景艳锋 ${ }^{a}$ 刘云云 $a, b \quad$ 万结平 $*, a, b$ \\ ( ${ }^{a}$ 江西师范大学化学化工学院 南昌 330022) \\ ( ${ }^{b}$ 教育部功能有机小分子重点实验室 江西师范大学 南昌 330022)
}

\begin{abstract}
摘要 缺电子烯胺结构上含有多个不同活性的亲核和亲电反应位点，同时双键本身可以作为烯键给予体参与反应，因 此可以通过各种亲核、亲电以及环加成等转化广泛应用于有机合成反应. 综述结合我们自己小组的研究工作和兴趣, 对基于缺电子烯胺如烯胺酮、烯胺酷等和含胺基试剂包括铵、胺、烯胺、艮和硫腿等之间的交叉氨化为关键转化, 并 和其它底物发生串联转化的多组分反应研究进展进行了总结.
\end{abstract}

关键词 缺电子烯胺; 交叉氨化; 多组分反应; 杂环

\section{Recent Advances on Multicomponent Reactions Based on the Transamination Process of Electron Deficient Enamines}

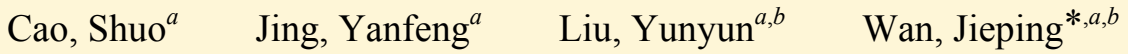 \\ ( ${ }^{a}$ College of Chemistry and Chemical Engineering, Jiangxi Normal University, Nanchang 330022) \\ $\left({ }^{b}\right.$ Key Laboratory of Functional Small Organic Molecules, Ministry of Education, Jiangxi Normal University, \\ Nanchang 330022)
}

\begin{abstract}
Electron deficient enamines bear several different nucleophilic and eletrophilic sites in their structures, they can also serve as donors of $\mathrm{C}=\mathrm{C}$ bonds in organic reactions. Therefore, electron deficient enamines have been broadly employed in organic synthesis via various nucleophilic, electrophilic transformation and cycloaddition. Based on the research interest and works of our group in the chemistry of electron deficient enamines, the research advances on multicomponent reactions (MCRs) are reviewed based on the transamination of electron deficient enamines with ammomium, amines, enamines, ureas and thioureas as key transformation.
\end{abstract}

Keywords electron deficient enamine; transamination; multicomponent reactions; heterocycle

烯胺是有机化学中最古老和经典有机化合物之一, 这类化合物通常可以由胺和含 $\alpha-$-碳的醛或酮缩合得到. 和普通的碳亲核试剂如醛酮 $(\alpha-$ - 碳)相比, 相应的烯胺具 有更高的 HOMO 轨道能量, 导致 $\alpha$-氢具有更强的酸性, 进而使得这类化合物具有更强的亲核性 ${ }^{[1,2]}$. 因此烯胺 类化合物不论是作为原料 ${ }^{[3 \sim 7]}$, 还是作为反应的活性中 间体 ${ }^{[8-12]}$, 在合成化合物中都显示了巨大的潜力. 当烯 胺 $\alpha$-碳和吸电子基团如羰基、酯基、硝基或氧基等相连 时, 得到的相应的烯胺酮、烯胺酯、硝基烯胺和氧基烯 胺都是典型的缺电子烯胺. 由于吸电子基团的存在, 双 键电子云进一步极化, 从而增加了和吸电子基团相连的 $\beta$-碳的亲电活性以及 $\alpha$-碳的亲核性. 此外, 当这类烯胺
中的氨基为 $\mathrm{NH}$ 或 $\mathrm{NH}_{2}$ 时, 氨基本身也是活泼的亲核位 点. 不仅如此, 烯胺中的双键在适当的条件下还可以作 为烯键给予体参与环加成反应. 因此，由于多个亲核、 亲电活性反应位点以及活泼双键的存在, 缺电子烯胺成 为有机合成化学中优秀且反应途径多样的合成砌块(图 $1)^{[13 \sim 16]}$.

在大量已报道的关于缺电子的烯胺参与的有机反 应中，根据参与反应关键位点的不同可以将反应进行归 类. 其中有一类重要的反应是通过以缺电子烯胺结构中 氨基的离去作为关键转化, 这些反应通常由亲核氨基试 剂和烯胺中的氨基发生形式上的交叉氨化反应 (transamination)引发, 然后原位生成的新烯胺类中间体,

*E-mail: wanjieping@jxnu.edu.cn

Received December 15, 2013; revised January 5, 2014; published online January 10, 2014.

Project supported by the National Natural Science Foundation of China (No. 21102059), the Project from the Department of Education of Jiangxi Province (No. GJJ13245) and the Sponsored Program for Cultivating Youths of Outstanding Ability in Jiangxi Normal University.

国家自然科学基金(No. 21102059)、江西省教育厅基金(No. GJJ13245)和江西师范大学青年英才计划资助项目. 


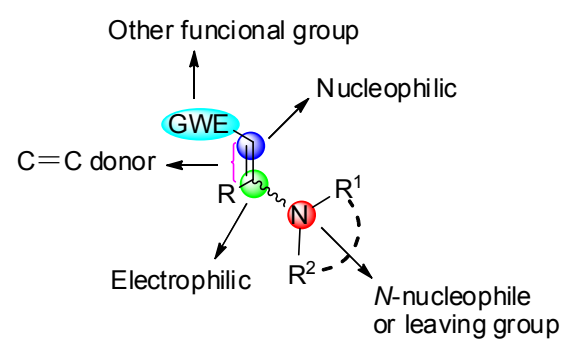

图 1 缺电子烯胺结构中的多反应活性位点

Figure 1 Versatile reactive sites in electron deficient enamines 再利用新烯胺中的活性反应位点和合适的原料通过串 联反应的方式继续转化. 当反应体系中存在除了缺电子 烯胺和含氨基的亲核试剂之外的第三个或更多反应原 料时, 反应通过多次化学键的形成或断裂, 以多组分反 应的方式产生各种不同类型的化合物. 有趣的是，尽管 交叉氨化这一转化在多种不同化学转化中都曾被发现 和应用 ${ }^{[17 ~ 20]}$, 对这一类型的反应目前尚无系统的总结. 因此, 我们结合自己的研究兴趣和近期相关工作, 对基 于缺电子烯胺中交叉氨化为关键转化引发的多组分反 应进行综述.

缺电子烯胺和含氨基的亲核试剂交叉氨化反应基 本过程如 Scheme 1 所示, 当氨基和缺电子双键相连时, 这类 $\mathrm{C}\left(\mathrm{sp}^{2}\right)-\mathrm{N}$ 化学键在相应的催化条件下和其它含氨 基的氮亲核试剂容易发生反应，原位生成 $\mathrm{N}$-取代的含 $\mathrm{NH}$ 基才的新缺电子烯胺, 这一中间体再利用新生成的 $\mathrm{NH}$ 亲核位点及这一缺电子体系中的其它活性位点和合 适的反应底物发生串联反应, 生成结构多样的多组分反 应产物. 基于一些相关文献报道和前期的研究结果, 我 们曾以典型的缺电子烯胺烯胺酮和伯胺之间的交叉氨 化为模型反应，对这一转化进行了初步的探索 ${ }^{[21]}$. 尽管 这一转化早期已有一些相关报道, 但主要都是在合成方 法中被作为中间体或辅助反应报道, 并没有对这一反应 过程进行系统研究的文献 ${ }^{[22,23]}$. 我们在对这一转化的探 索中, 首先对反应催化条件进行了篮选, 发现以水作为 溶剂时, 不论是 Lewis 酸如 $\mathrm{FeCl}_{3}, \mathrm{AlCl}_{3}, \mathrm{FeSO}_{4}, \mathrm{TMSCl}$, CAN(硝酸铈胺), 还是 Brønsted 酸如乙酸等都能很好地 催化这一转化. 同时, 我们在此基础上进一步通过考察 具有不同官能团的烯胺酩和伯胺之间的反应, 证实了这 一转化对各类不同官能团的优秀耐受性. 更重要的是, 通过以重水作为溶剂进行同位素标记实验, 我们发现烯 胺酤 1 和伯胺 2 反应得到的主要产物为忥标记的 NH 烯 胺酮 3-d. 基于这一发现, 我们提出了这一转化的可能 机理(Scheme 2), 即反应大体经历了加成-消除的转化 过程. 首先是烯胺醖中的双键和水加成得到㲴标记的加 成中间体 4, 这一中间体经历脱水原位转化成 $\beta$-羰基醛 中间体 $\mathbf{5}$, 而 5 很容易经过中间体 $\mathbf{6}$ 再和伯胺缩合并异
构化得到最终在形式上发生了交叉氨化的烯胺酮产物 3. 这一可能的反应机理也可以解释大部分基于这类交 叉氨化过程的反应. 本文即对基于缺电子烯胺的不同类 型交叉氨化为关键转化的多组分反应研究进展进行总 结和综述.

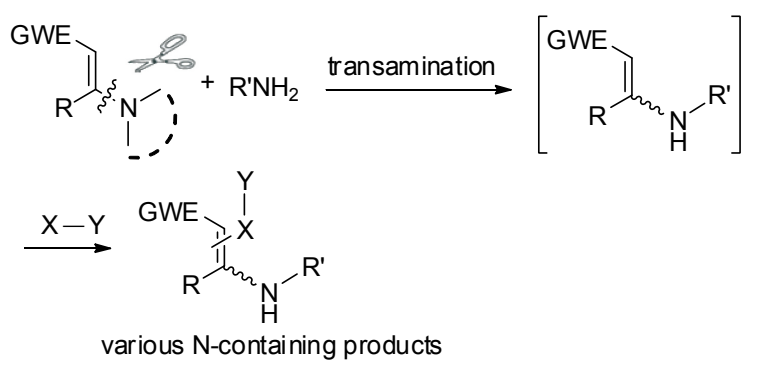

Scheme 1
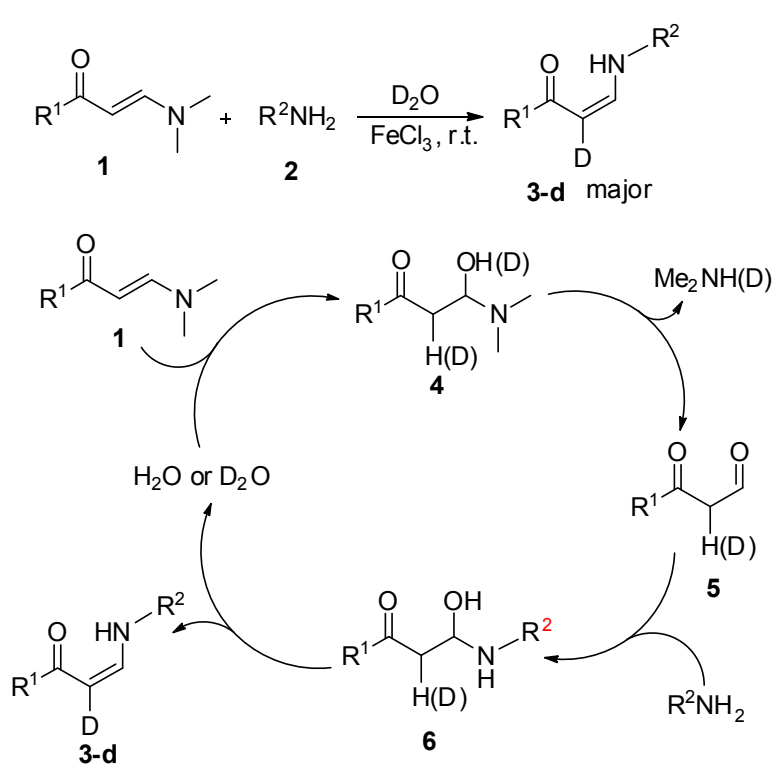

Scheme 2

\section{1 基于铵亲核试剂交叉氨化的多组分反应}

较早出现的基于缺电子烯胺的交叉氨化的多组分 反应是通过烯胺酮、1,3-二羰基化合物和醋酸铵三组分 合成吡啶的反应. 反应所使用关键原料之一是 $N, N-$ 二甲 基取代的烯胺酮 1 , 这类化合物主要的合成方法是通过 相应的酩和 $N, N$-二甲基甲酰胺二甲基缩醛(DMF-DMA) 在非极性溶剂如二甲苯等中加热回流来合成，具体可参 见 $\mathrm{Li}$ 等 ${ }^{[14]}$ 综述中的详细介绍, 同时, 基于烯胺酮的典型 反应在该综述中也有系统的总结, 本文将集中对相关的 基于交叉氨化的多组分反应进行综述. Elnagdi 等 ${ }^{[24]}$ 在 研究烯胺酤这类典型缺电子烯胺的过程中发现在回流 乙酸的条件下, 烯胺酥先和 1,3-二羰基化合物发生 Michael 加成得到中间体 $\mathbf{8}$, 而中间体 $\mathbf{8}$ 中的二甲氨基通 过和铵的原位交叉氨化以及分子内缩合脱水以及氧化 
会生成吡啶产物 7. 这一转化利用了交叉氨化得到的亲 核 $\mathrm{NH}_{2}$ 基团和烯胺酮结构中的亲核羰基, 但是这一工作 当时并没有系统展开(Scheme 3). 然而, 这一高选择性 合成 1,2,6-三取代吡啶的方法在最近得到了关注, 不同 的改进催化体系被发现并报道用于系统合成这类吡啶 衍生物. Reddy 等 ${ }^{[25]}$ 以 montmorillonite $\mathrm{K} 10$ 作为固体酸 催化剂, 在更温和的异丙醇回流条件下实现了同样的反 应, 这一方法的另一个优势是产物可直接通过重结晶进 行提纯(Scheme 4). 随后, Kantevari 等 ${ }^{[26]}$ 采用杂多酸 $\mathrm{K}_{5} \mathrm{CoW}_{12} \mathrm{O}_{40} \cdot 3 \mathrm{H}_{2} \mathrm{O}$ 为催化剂更系统地对这一反应进行 了研究, 发现这一杂多酸催化剂在异丙醇回流或无溶剂 加热 $\left(115{ }^{\circ} \mathrm{C}\right)$ 条件下均能高效催化这一三组分合成吡啶 的反应. 反应不仅适用于链状 1,3-二羰基化合物, 也适 用于环状 1,3-二酮如达米酮合成相应的吡定稠环衍生 物, 反应结束后, 杂多酸催化剂可以回收并重新用于催 化反应(Scheme 4). 这一研究小组 ${ }^{[27]}$ 随后又报道了微波 辅助条件下同一杂多酸催化的这类反应，类似的吡啶衍 生物可以在几分钟时间内合成. 最近, 这一课题组 ${ }^{[28]}$ 又 采用 $\mathrm{CeCl}_{3} \cdot 7 \mathrm{H}_{2} \mathrm{O}-\mathrm{NaI}$ 的催化体系在回流异丙醇条件下 对含有多种芳环、芳杂环以及多环结构 $\left(\mathrm{R}^{1}\right)$ 的烯胺酮参与 的和 1,3-二羰基化合物及醋酸铵的三组分反应，合成了 48 个含有不同芳基骨架的吡啶类化合物 7, 并对这些化 合物的抗分支杆菌活性进行了测试, 发现了具有较好活 性的先导化合物. 在对这个三组反应合成吡啶方法研究 的基础上, Kantevari 小组 ${ }^{[29]}$ 进一步对含有其它官能结构 的烯胺酮类化合物参与的相应吡啶衍生物合成反应进行 了研究, 例如: 他们合成了具有二氢苯并呋喃结构的烯 胺酮 10, 并采用 $\mathrm{CeCl}_{3} \cdot 7 \mathrm{H}_{2} \mathrm{O}-\mathrm{NaI}$ 和异丙醇回流的催化体 系, 以 10 和不同的 1,3-二羰基化合物及醋酸铵反应, 合 成了一系列在 6 位含有苯并二氢呋喃结构的相应吡啶衍 生物 11 (Eq. 1). 这一三组分方法学同时还被扩展应用到

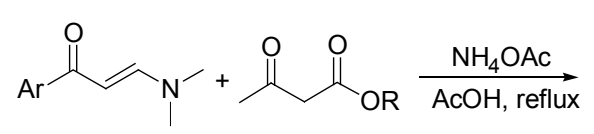<smiles>[R]OC(=O)C(C(=O)C(C)C)C(CC(=O)Br)(N(C)C)N(C)C</smiles>

Scheme 3

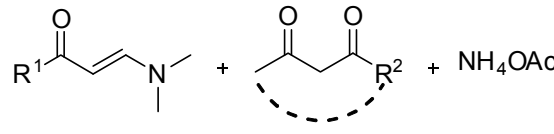

montmorillonite $\mathrm{K} 10$ $i$-PrOH, reflux

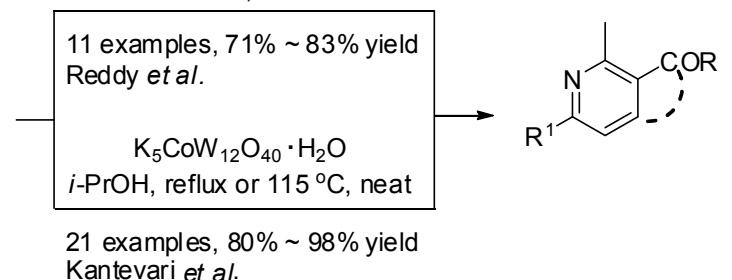

Scheme 4

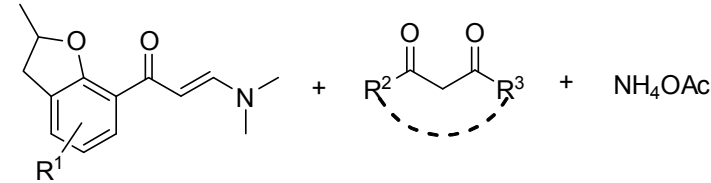

10

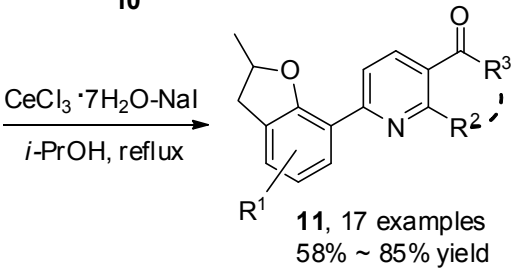

$\mathrm{R}^{2}=$ alkyl, $\mathrm{R}^{3}=$ alkyl or alkoxy

了含有核苷片段的吡啶衍生物中. 方法是先合成含非环 状核苷片段的烯胺酮 12，并以此为起始物和 1,3-二羰基 底物以及醋酸铵进行三组分反应，同样在回流异丙醇中 以 $\mathrm{CeCl}_{3} \cdot 7 \mathrm{H}_{2} \mathrm{O}-\mathrm{NaI}$ 为催化剂, 通过核甘苷烯胺酮 $\mathbf{1 2}$ 上的 交叉氨化为关键转化, 再经过串联转化得到相应的含非 环状核苷结构的吡定衍生物 13 (Eq. 2) ${ }^{[30]}$.

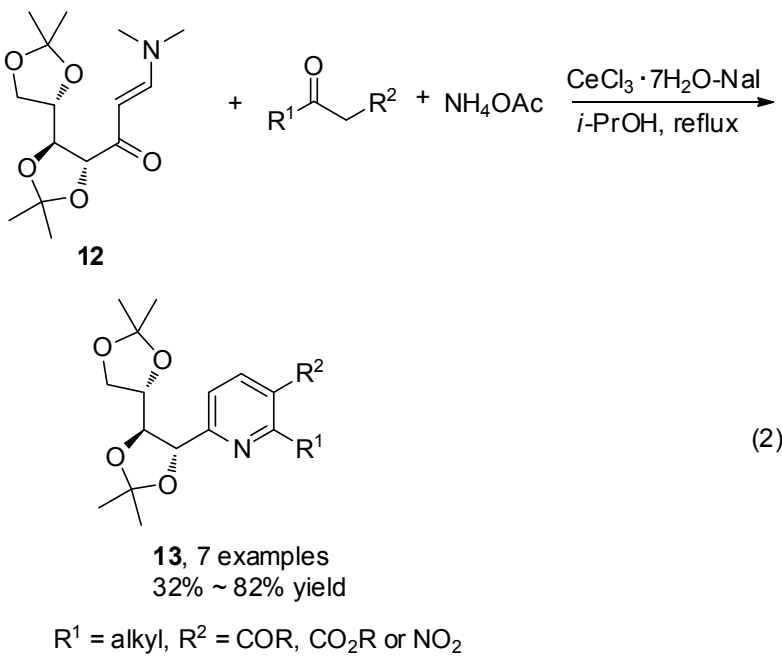

在大多数情况下，这类基于烯胺酮交叉氨化的三组 分反应都采用含 1,3-二羰基结构的活泼亚甲基类化合物 
作为另一主要原料, 合成得到的产物在结构多样性上受 到一定限制. 因此, 发展更有效的催化体系对这一参与 反应的组分进行扩展也引起了关注. Siddiqui 等 ${ }^{[31}$ 通过 采用纳米氧化锌作为催化剂, 成功实现了烯胺酮、醋酸 铵和一系列不同类型的具有活泼亚甲基(甲基)底物如 $\beta$ 羰基酯、链状 1,3-二酮、含杂原子 1,3-环二酮、4-羊基 香豆素以及环单酮的三组分反应, 分别合成了相应的吡 啶衍生物 7, 14, 15, 16 和 17, 大大拓展了这一多组分反 应在合成多样性分子上的应用范围, 反应在无溶剂、70 ${ }^{\circ} \mathrm{C}$ 加热条件下即可高效进行, 且纳米氧化锌催化剂可以 回收重复利用, 且催化活性不受明显影响(Scheme 5).

\section{2 基于胺亲核试剂交叉氨化的多组分反应}

作为优秀亲核试剂, 胺类有机物在有机合成中具有 非常多样和广泛的应用. 我们研究发现对于含 $N, N^{\prime}$-双 取代的缺电子烯胺, 以伯胺作为胺亲核试剂时交叉氨化 反应容易发生(Scheme 2). 而且由于伯胺在母体结构上 的灵活可变性, 基于这类交叉氨化的反应在合成化合物 结构多样性方面具有巨大的优势. 2009 年, Wan 等 ${ }^{[32]}$ 报 道了烯胺酮 1 , 伯胺和 $\alpha, \beta$-不饱和醛 18 的三组分合成吡 啶的反应. 这一反应根据胺底物的不同可以分别生成 1,4-二氢吡啶 19 (1,4-DHPs) 和 1,2-二氢吡啶 20 (1,2-
DHPs)为主要或唯一产物. 通过对反应体系中的生成物 分离分析以及控制对比反应，发现交叉氨化中间体烯胺 酮 3 的生成是反应的关键转化步骤. 如 Scheme 6 所示, 起始原料烯胺酮 $\mathbf{1}$ 和伯胺 $\mathbf{2}$ 发生快速交叉氨化生成中间 体 3 后，根据该中间体中和 $\mathrm{NH}$ 相连的烃基性质(电子和 位阻效应), 可以分别通过 $\mathrm{NH}$ 作为第一亲核位点进攻 $\alpha, \beta$-不饱和醛的醛基(path a)或以中间体 $\mathbf{3}$ 中的亲核碳为 第一亲核位点进攻醛基(path $b, R^{2}$ 为强吸电子或大位阻 基团, $\mathrm{NH}$ 的亲核作用被削弱). 两种途径转化分别得到 中间体 21 和 22, 并进一步发生分子内关环得到咕二氢 吡啶产物 19 和 20 . 随后, Wan 等 ${ }^{[33]}$ 又尝试用烯胺酮、伯 胺、醛和硫脲进行四组分反应, 在乙醇为介质室温反应 的温和条件下, 通过三甲基氯硅烷( TMSCl)和硝酸铈铵 $(\mathrm{CAN})$ 为混合催化体系, 成功实现了四氢嘧啶硫酮类化 合物 23 的非对映选择性合成, 产物结构含 3 个手性中 心, 但只分离到一对对映体产物, 反应也经历了胺和烯 胺酩中二甲胺基交叉氨化过程. 值得一提的是这类合成 在以经典的 1,3-二羰基类化合物替代烯胺酮时，类似的 反应无法实现，反应可能是通过先交叉氨化得到中间体 3, 再和醛以及硫脲反应, 也可能是先生成二甲氨基取 代的四氢嘧啶䣶中间体 23', 这一中间体再和芳胺发生 交叉氨化得到目标产物 23 (Scheme 7).<smiles>[R]OC(=O)c1ccc([Y])nc1C</smiles>

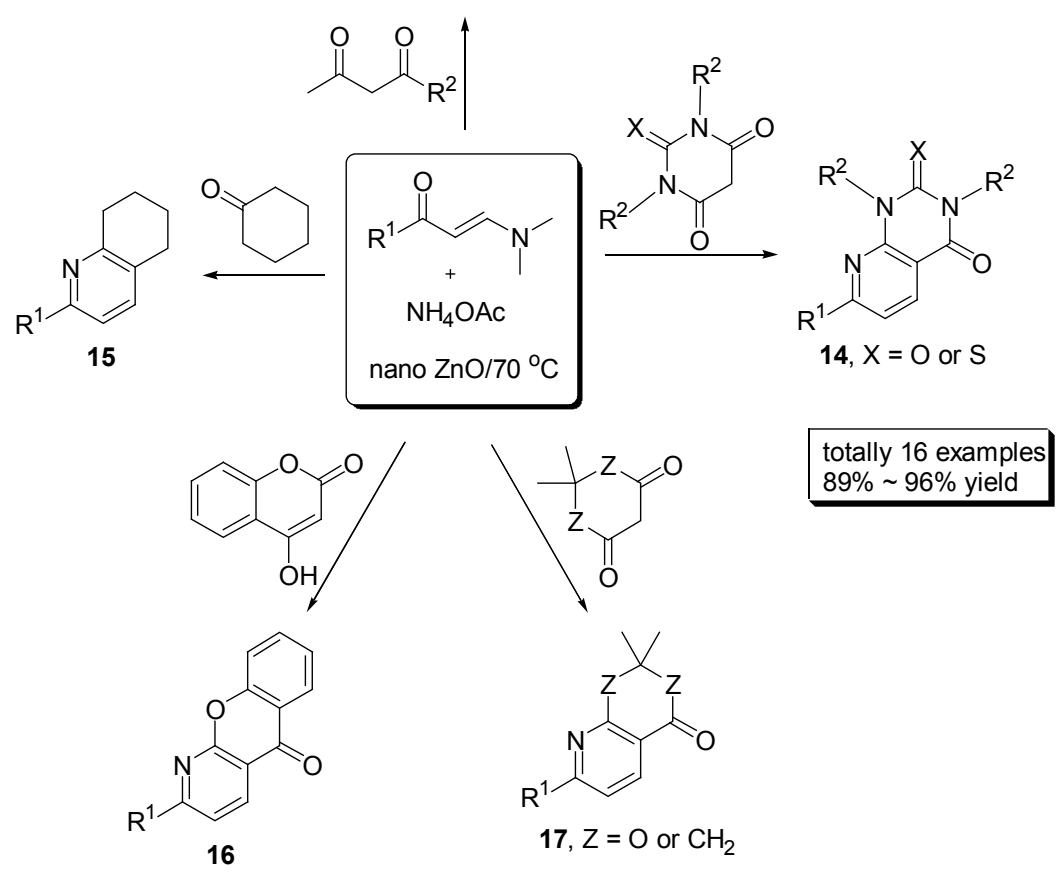

Scheme 5 
<smiles>[R][R]1ccc(/C=C/C=O)cc1</smiles>

19, 25 examples $14 \% \sim 83 \%$ yield<smiles>[R]C(=O)C1=CN([R])C(c2ccccc2)C=C1</smiles>

20, 7 examples $51 \% \sim 63 \%$ yield

$\mathrm{OH} \mathrm{O}$<smiles>[R16][R9]</smiles>

Scheme 6

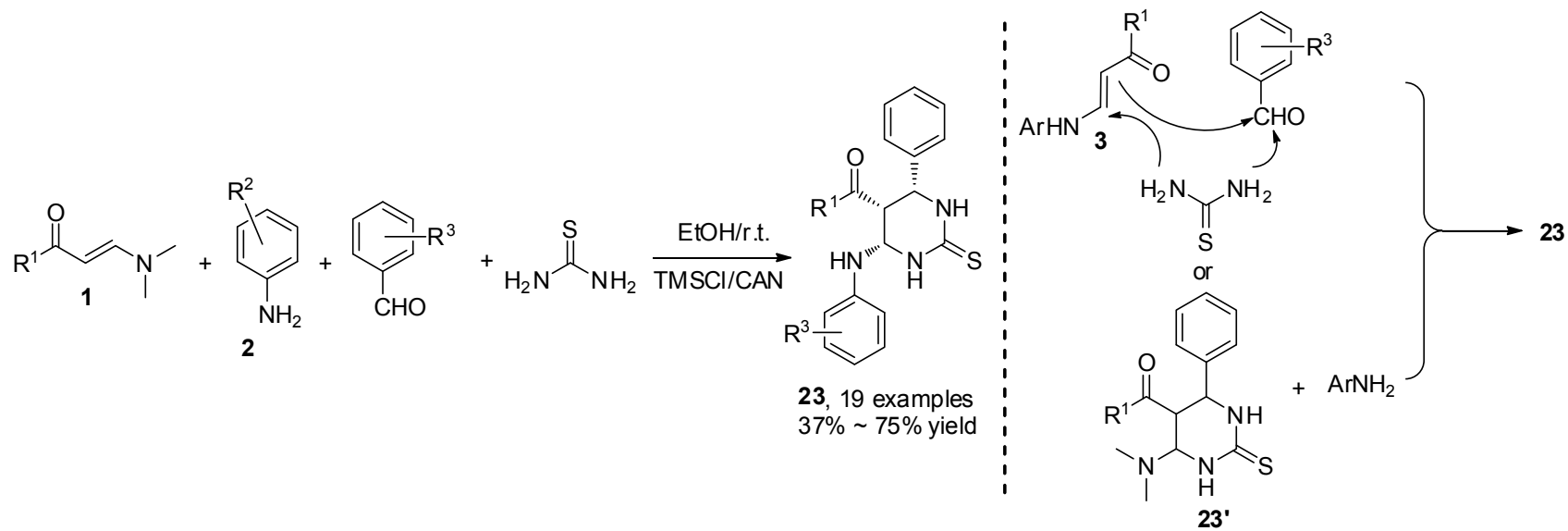

Scheme 7

Perumal 等 ${ }^{[34]}$ 以这类缺电子烯胺为底物, 和芳基伯 胺、甲醛一起作为原料, 在 DMF 为溶剂 $120{ }^{\circ} \mathrm{C}$ 加热条 件下, 通过对甲醛用量的优化调整, 实现了 1,3-二芳基 六氢嘧啶类化合物 25 的合成. 这一反应首先通过烯胺 酮 1 和芳基伯胺的交叉氨化以及两次脱水关环得到二氢 嘧啶中间体 24, 24 在过量的甲醛存在下原位被还原则得 到产物 25. 这一反应通过利用甲醛作为亲电试剂和还 原剂的双重性质, 以缺电子烯胺的交叉氨化为关键转 化, 建立了一种简便的合成六氢嘧啶的方法(Scheme 8).

同样以烯胺酥为主要底物之一, Wan 等最近发展了 一类合成具有对称结构的 2,6-未取代 1,4-二氢吡啶 23 的 方法. 这一方法适用合成各种含 $N$-芳基、 $N$-烷基结构的 二氢吡啶，同时也适用于含芳基和烷基 $\left(\mathrm{R}^{1}\right)$ 取代的烯胺 酮. 更为有趣的是, 交叉氨化过程不仅在这一多组分

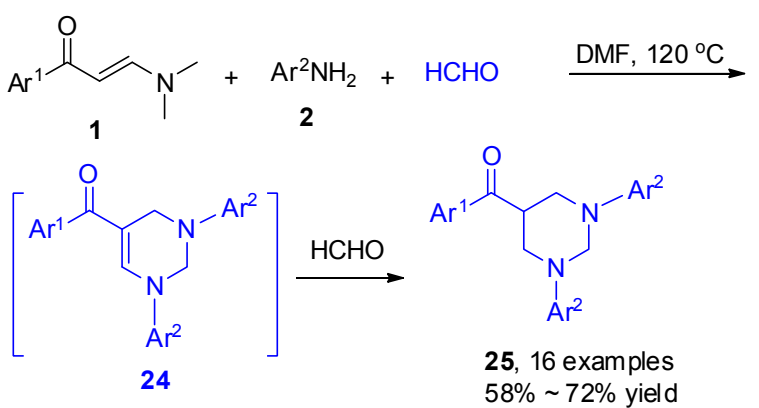

Scheme 8

合成方法产生关键中间体 3 , 同时还通过交叉氨化在反 应过程中产生一个 “副产物-中间体一产物” 的原子经济 性循环过程, 使得副产物都转化为目标产物. 具体过程 如 Scheme 9 所示, 烯胺酩和伯胺交叉氨化得到中间体 
3, 两分子的 3 和醛环化得到目标产物 1,4-二氢吡啶 23 的同时, 产生等物质的量的伯胺副产物, 而在催化条件 下, 伯胺和烯胺酩原料 1 又循环重复交叉氨化过程, 得 到关键中间体 3 , 并进一步转化生成目标产物 23, 通过 不断循环, 最终可以避免伯胺副产物的生成 ${ }^{[35]}$. 这一反 应通过调整产物, 即加入和醛等物质的量 1,3-二酮 24 作 为其中一等物质的量的烯胺酮替代原料, 可以相应地通 过交叉氨化的关键转化合成得到具有并环取代结构的 1,4-二氢吡啶(四氢喹诺酤)产物 25. 这一合成方法最近 由 Perumal 等 ${ }^{[36]}$ 以 $\mathrm{AcOH}$ 为溶剂, $120{ }^{\circ} \mathrm{C}$ 加热为反应条 件得以实现(Eq. 3). 最近, Perumal 等 ${ }^{[37]}$ 又利用水合茚三 酮为原料, 和烯胺酮 1 以及芳香伯胺 $\mathbf{2}$ 为原料, 实现了 三组分合成茚酩并二氢吡咯衍生物的反应. 反应在醋
酸存在的条件下通过室温研磨 5 8 min 可以完成. 如 Scheme 10 所示，反应也通过交叉氨化得到的中间体 3 进行，中间体 3 结构中的亲核氨基和亲核 $\beta$-碳分别进攻 狮三酮 1 和 2 号碳上的羰基，通过缩合脱水和类 aldol 反应串联转化得到目标产物狮酮并二氢吡咯 26 , 反应 对胺和烯胺酮的容忍性较好, 产率优秀. 值得一提的是 反应的区域选择性优秀, 以亲核氨基和亲核 $\beta$-碳和狮三 酮发生相反位点亲核环化的产物在反应中没有发现.

\section{3 基于缺电子烯胺亲核试剂交叉氨化的多组分 反应}

在涉及缺电子烯胺交叉氨化转化的有机反应中，除 了常见的氨基亲核试剂如铵盐和胺类化合物常用作亲

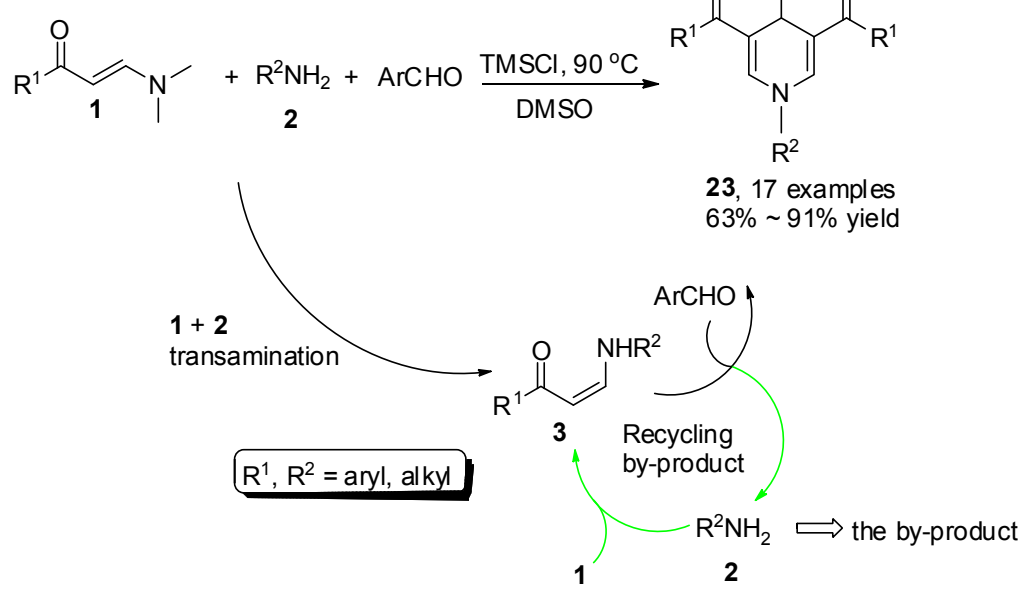

Scheme 9
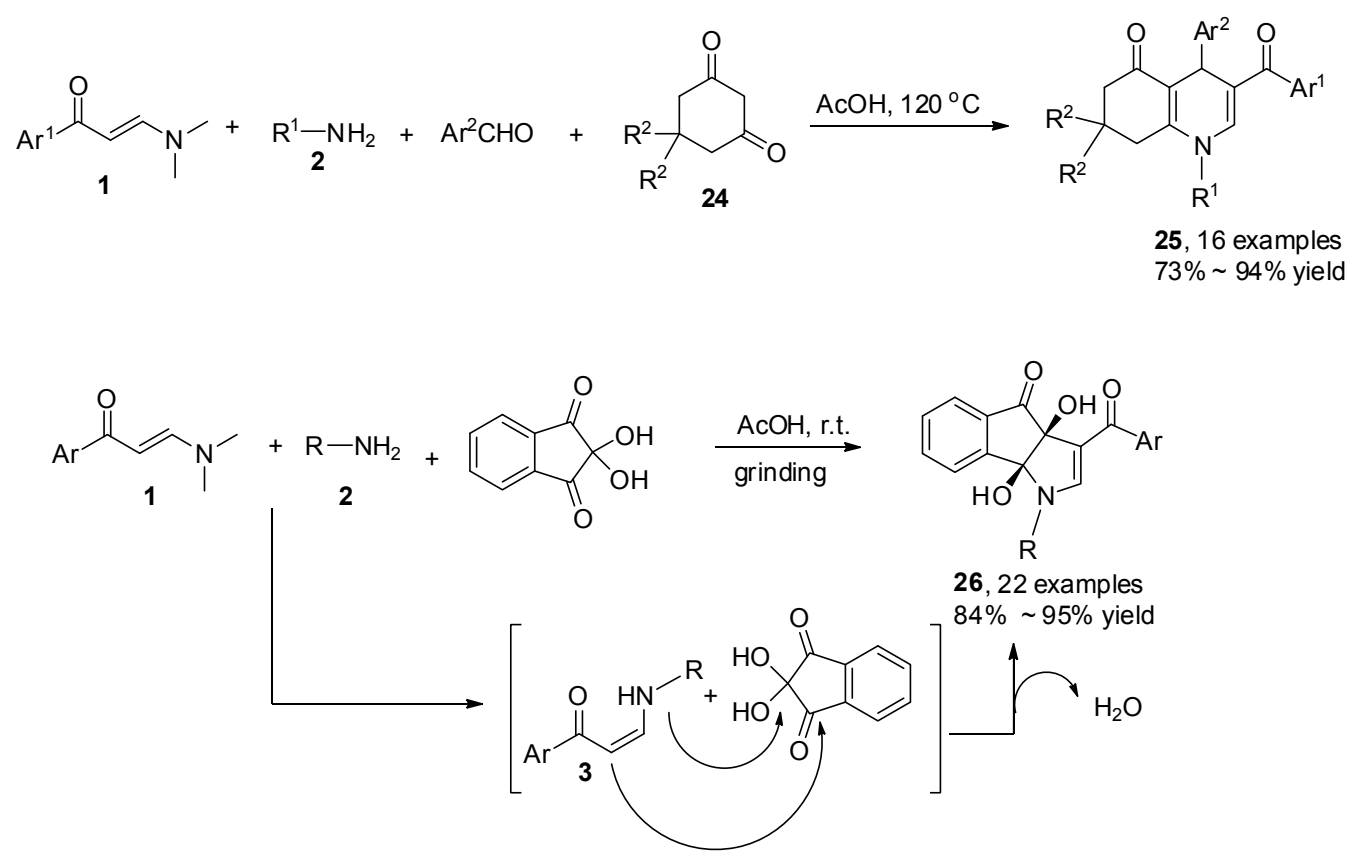

\section{Scheme 10}


核试剂和缺电子烯胺发生交叉氨化反应, 缺电子烯胺本 身因为含有亲核氨基的结构, 在适合的条件下, 相互之 间也可以发生交叉氨化反应, 通过氨化后得到的活性中 间体继续转化生成结构多样的有机化合物. 这一来反应 早在 1946 年已经被用于合成杂环化合物, Petrow ${ }^{[38]}$ 以两 种缺电子的烯胺 28 ( $\beta$-氨基脂)和 29 ( $\beta$-氨基氰) 和邻硝基 苯甲醛 27 在回流乙醇条件下反应得到了 1,4-二氢吡啶 30, 并通过 30 合成了多种含氮杂环化合物, 但反应需要 很长的时间, 且产率较低(Eq. 4), 最近, Mirza-Aghayan 等 ${ }^{\left[{ }^{39]}\right.}$ 采用 2 倍物质的量的 $\beta$-氨基甲酯 31 和醛作为起始 物, 通过三甲基氯硅烷催化或 $\mathrm{MeI} /$ 三乙基硅烷 $/ \mathrm{PdCl}_{2}$ 催 化体系实现了具有对称结构的 1,4-二氢吡啶 32 的合成, 这一三组分反应也是通过交叉氨化转化实现的, 且交叉 氨化发生在两分子的氨基酯 31 之间(Reaction 1, Scheme 11). 之后, $\mathrm{Li}$ 等 ${ }^{[00]}$ 直接采用含 $\mathrm{NH}$ 的烯胺酮 3 为起始原 料, 通过类似的交叉氨化一环化过程和醛反应实现了 $N$ 取代的对称 1,4-二氢吡啶 33 的多组分合成, 反应在苯磺 酸或者氯金酸钠催化条件下进行, 使用与芳香族伯胺和 脂肪族伯胺以及含芳基和烷基的烯胺酮 (Reaction 2, Scheme 11), 这一反应的关键中间体 Arylidene bis(3arylaminoacrylates)在作者的研究和相关文献中都被成 功合成分离, 并确证了结构 ${ }^{[41]}$.

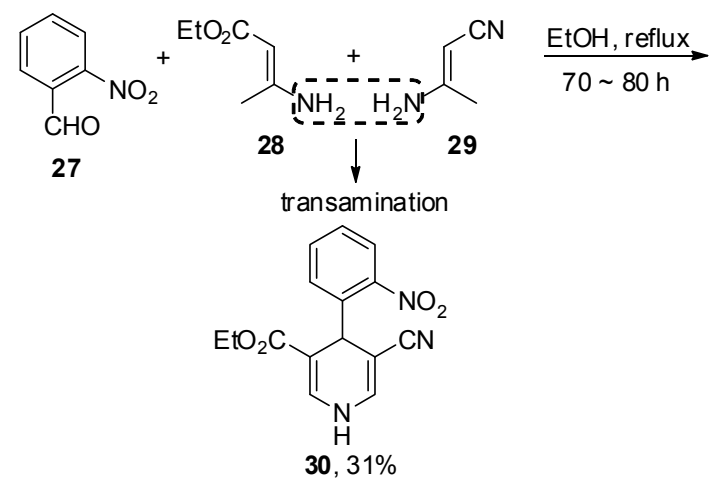

另一个基于 $\beta$-氨基酯 3 这类缺电子烯胺的多组分反 应是这一化合物本身的三聚反应，Ajavakom 等 ${ }^{[42]}$ 发现 在 $\mathrm{TiCl}_{4}$ 的催化下, $\mathbf{3}$ 在室温条件下可以发生自身的聚合 反应生成 4 位含有羧酸酯取代的 1,4-二氢吡啶产物 34 . 在这一反应过程中，其中一分子的氨基酯作为亲电试 剂，和另一分子氨基酯先发生二聚反应，得到含共轭二 烯结构的中间体 35,35 在催化条件下被第三分子的氨基 酯以 Michael 加成的方式进攻，得到三聚中间体 36. 最 终，中间体 36 通过分子内交叉氨化反应脱去一分子胺, 并同时生成目标产物 34 (Scheme 12). 利用含有 $\mathrm{NH}_{2}$ 基 团的缺电烯胺分子间的交叉氨化作用也可以合成吡啶 衍生物. 例如, 2 倍物质的量的烯胺 $37\left(\mathrm{R}^{1}=\right.$ hetero aryl, $\mathrm{CO}_{2} \mathrm{Et}$ or $\left.\mathrm{CN}\right)$ 和原甲酸三乙酯在 $\mathrm{Hf}(\mathrm{OTf})_{4}$ 的催化作用下 可以直接合成得到吡啶衍生物 39 , 反应首先也是通过 交叉氨化的方式生成中间体 $\mathbf{3 8}, \mathbf{3 8}$ 对原甲酸三乙酯进行 两次亲核取代反应环化后再消除一分子乙醇就可以得 到吡啶产物 39. 由于不需要经历脱氢过程，反应在无氧 化剂存在条件即可发生(Scheme 13) ${ }^{[43]}$.

\section{4 基于腿/硫嫝核试剂交叉氨化的多组分反应}

由于亲核性相对较强, 目前胺、烯胺和铵盐是缺电 子烯胺交叉氨化反应中使用的主要氨基亲核试剂. 而其 它含氨基的亲核试剂如酰胺、艮、肼、羟胺等引发交叉 氨化转化的反应则相对较为少见. 目前已知的代表性多 组分反应为脲/硫䐖、醛和缺电子烯胺的三组分合成 3,4二氢嘧啶(硫)酮的反应. 在经典合成化学中，以脲、硫 脲、醛和 1,3-二羰基化合物的三组分 Biginelli 反应合成 这类化合物最为常见且为人们熟知, 为进一步扩大合成 这类具有重要生物活性的杂环化合物的结构多样性，设 计和发展其它多组分合成方法也一直是合成化学研究 的主要内容 ${ }^{[4]}$. Wan 等利用烯胺酮 1 为关键原料, 在
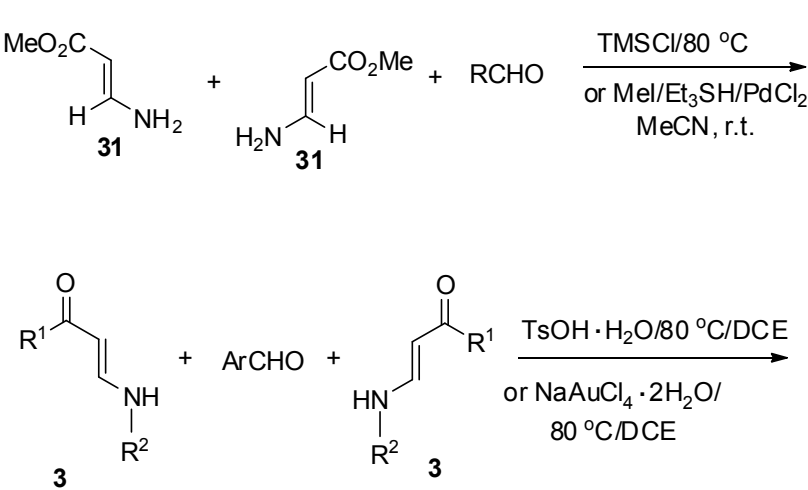<smiles>[R]C1C(C(C)=O)=CNC=C1C(C)=O</smiles>
$65 \% \sim 95 \%$ yield<smiles>[R]OC1=CN([R])C=C(C([R])[R])C1[Al]</smiles>

33, 14 examples $48 \% \sim 90 \%$ yield
(1)

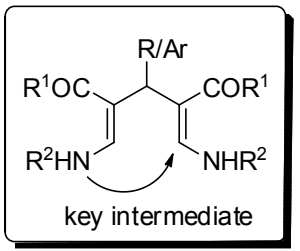

(2)

Chin. J. Org. Chem. 2014, 34, 876 885 


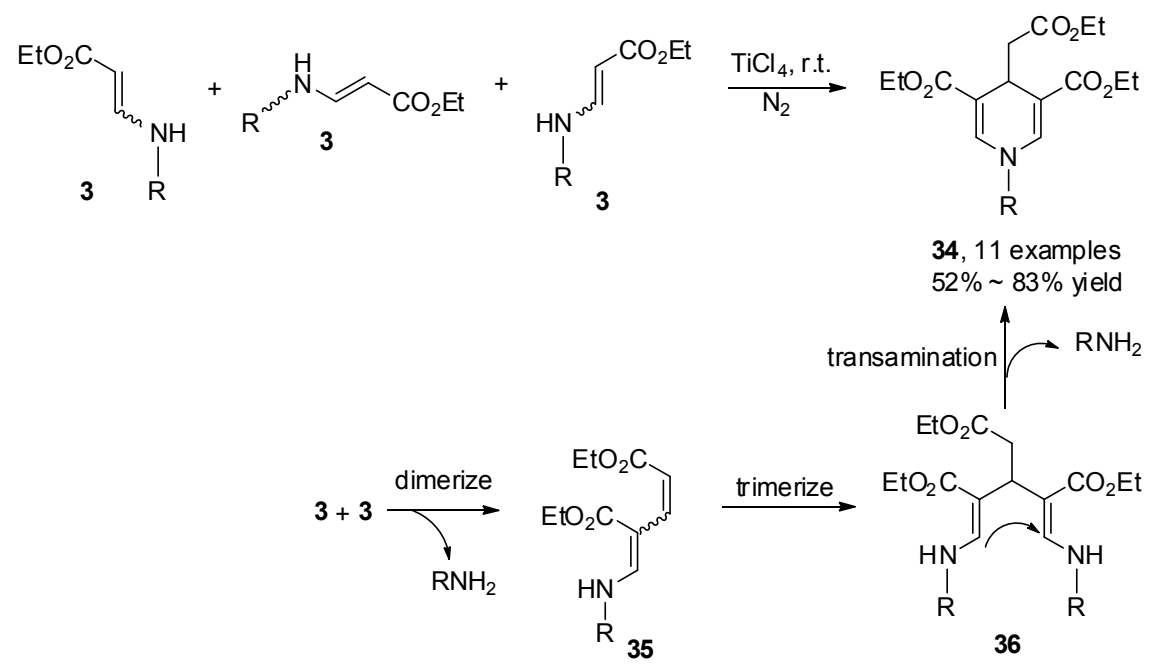

Scheme 12

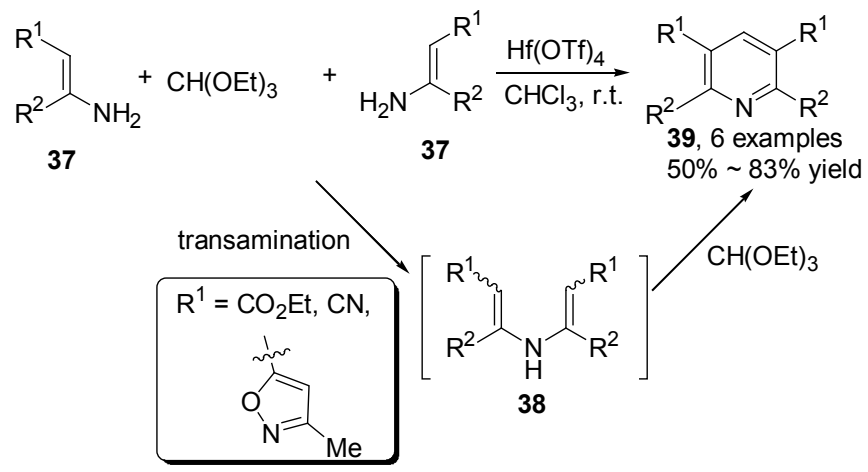

Scheme 13

$\mathrm{TMSCl}$ 为促进剂的条件下, 和醛和脲/硫脲进行三组分 反应, 成功合成了一系列结构多样的 3,4-二氢嘧啶(硫) 酮 41, 这一合成方法对这类化合物的合成具有优秀的 应用范围, 包括脂肪醛、芳香醛、嫝、硫嫝以及含多种 取代基的芳基烯胺酮都能发生这一反应, 并以良好到优 秀的产率得到目标产物. 有趣的是, 对于一些含特定官 能团的底物, 这一反应表现出了不一样的化学和区域选 择性. 例如, 当 $N$-甲基取代的硫脲用于这一反应时, 得 到的主要产物是 1,3-噻嗪类化合物 42, 这一结果可能是 因为 $N$-甲基的位阻作用使得该氨基难以进攻烯胺酮上 的二甲氨基发生交叉氨化, 而此时亲核性更强的硫原子 作为亲核试剂进攻烯胺酩, 将二甲氨基置换消除得到产 物 42. 当以 2-羊基苯基烯胺酮作为底物时, 反应表现出 更不一样的选择性，直接通过烯胺酮分子内羟基和二甲 氨基的亲核交换过程关环，再和醛以及氨基亲核试剂 (脲、硫脲或酰胺)发生类似 Mannich 的三组分反应, 得 到色酮衍生物 43 , 当嫝或硫脲作为底物时, 两次缩合的 产物 44 有时作为副产物出现(Scheme 14). 这些不同选 择性反应的出现不仅提供了合成不同类型化合物的简 便方法, 更重要的是证实了缺电子烯胺酮中的氨基可以
和含氧、硫的亲核试剂发生类似交叉氨化的转化，为合 成其它含氧、硫杂环的化合物提供了线索 ${ }^{[45]}$. 在这一工 作报道后不久, Abdelhamid 等 ${ }^{[46]}$ 以醋酸/dioxane 体系进 行了类似的反应，也合成得到结构类似的 2,4-二氢嘧啶 䣶产物, 但反应的适用范围较窄, 例如硫嫝不能进行类 似的反应，产物产率中等(Eq. 5).

\section{5 结论}

作为有机化学中经典活性反应结构之一, 对烯胺化 学的研究历史已经超过 100 年, 由于烯胺比传统的碳亲 核试剂有更好的亲核活性，同时又具有比很多反应活性 中间体相对更好的稳定性，烯胺在合成化学中的既可以 直接作为反应原料应用于有机合成, 同时也是许多有机 反应的关键中间体, 因而烯胺和合成和应用在有机合成 中一直占据重要地位 ${ }^{[47]}$. 而缺电子的烯胺不仅具有经 典烯胺的主要优点, 同时由于新官能团或结构片段的引 入，使得这类化合物设计相应的有机合成反应上具有更 大的灵活性. 许多缺电子的烯胺类化合物如烯胺酮、烯 胺酯等在有机合成化学中已经得到非常广泛的应用, 特 别是在多组分反应合成多样结构的有机小分子方面, 这 


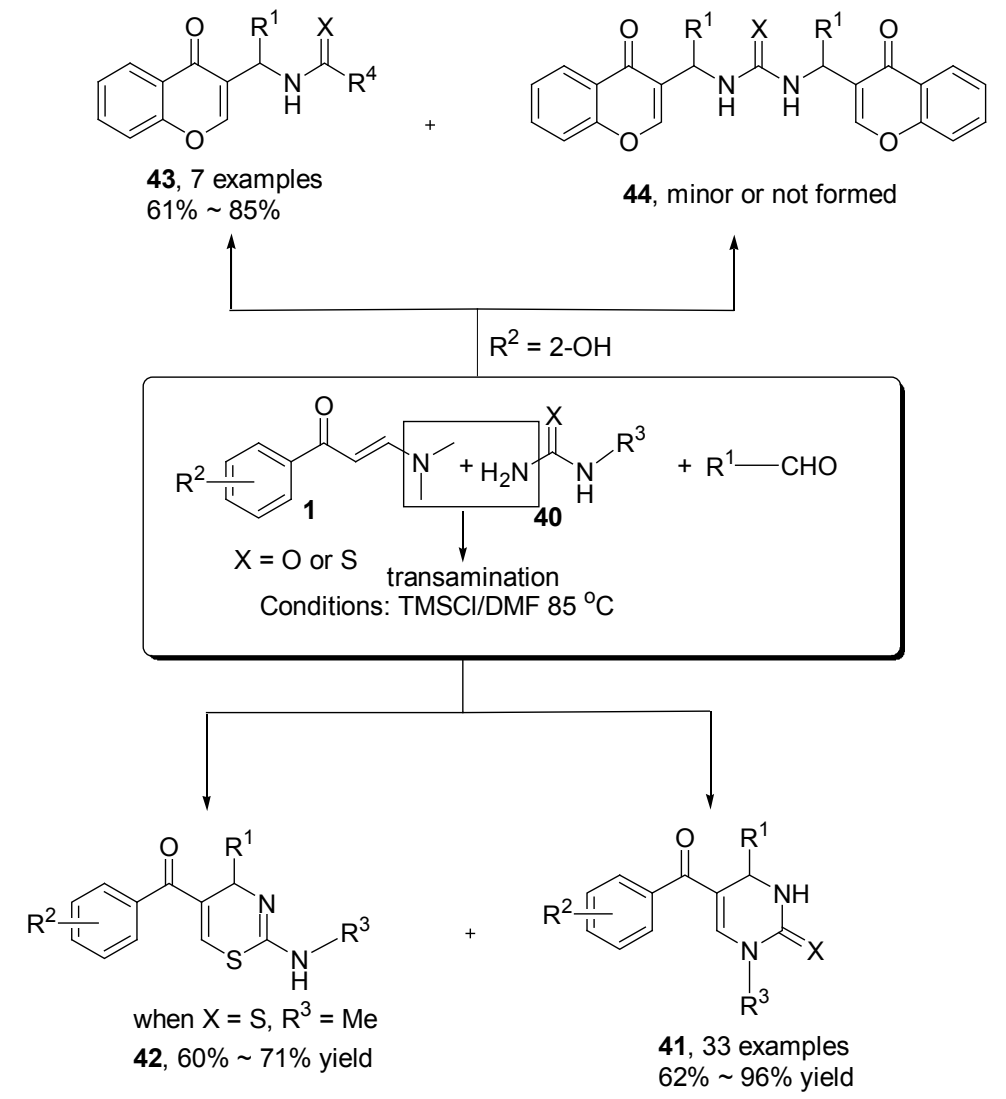

Scheme 14

$\mathrm{Ar}_{1}^{2} \overbrace{40}^{\mathrm{O}} \mathrm{N}_{\mathrm{N}}^{-}+\mathrm{H}_{2} \mathrm{~N}_{\mathrm{NH}_{2}}^{\mathrm{O}}+\mathrm{Ar}^{1}-\mathrm{CHO} \frac{\mathrm{AcOH}}{\text { dioxane, reflux }}$<smiles>O=C1NC=C(C(=O)[AlH2])C([Al])N1</smiles>

41, 6 examples $63 \% \sim 70 \%$ yield

类化合物因为具有多个不同的反应位点而具有独特的 优势. 本文结合我们自己的研究兴趣, 通过对烯胺酮和 不同胺基亲核试剂的交叉氨化这一关键转化, 对相关的 研究进展进行了系统的总结. 通过这些结果, 一方面可 以发现交叉氨化转化在相关多组分合成中的重要作用, 在合成目标化合物如 1,4-二氢吡啶等过程中途径多样; 而另一方面，目前的相关结果也反应出该研究领域面临 的挑战, 例如合成杂环化合物的母体结构多样性比较有 限, 主要合成的目标产物还是吡啶、嘧啶类衍生物, 而 且交叉氨化所涉及的氨基亲核试剂的类型也有待扩展, 特别是亲核性相对较弱的氨基亲核试剂如酰胺等还没 有能发生类似交叉氨化反应报道. 因此, 更系统地研究
这类转化并用于功能有机化合物的多样性合成仍然是 合成化学研究的重要内容.

\section{References}

[1] Mukherjee, S.; Yang, J. W.; Hoffmann, S.; List, B. Chem. Rev. 2007, 107, 5471 .

[2] Grondal, C.; Jeanty, M.; Enders, D. Nat. Chem. 2010, 3, 167.

[3] Kuehne, M. E. J. Am. Chem. Soc. 1959, 81, 5400.

[4] Malhotra, S. K.; Hostynek, J. J.; Lundin, A. F. J. Am. Chem. Soc. 1968, 90, 6565.

[5] Hayakawa, Y.; Yokoyama, K.; Noyori, R. J. Am. Chem. Soc. 1978, 100, 1799.

[6] Koradin, C.; Gommermann, N.; Polborn, K.; Knochel, P. Chem. Eur. J. 2003, 9, 2797.

[7] Koradin, C.; Polborn, K.; Knochel, P. Angew. Chem., Int. Ed. 2002, $41,2535$.

[8] Enders, D.; Huttl, M. R. M.; Grondal, C.; Raabe, G. Nature 2006 $441,861$.

[9] List, B. Acc. Chem. Res. 2004, 37, 548.

[10] Han, J.; Paton, R. S.; Xu, B.; Hammond, G. B. Synthesis 2013, 463.

[11] Xu, D.-Q.; Xia, H.-B.; Luo, S.-P.; Tang, J.; Zhang, S.; Jiang, J.-R.; Xu, Z.-Y. Angew. Chem., Int. Ed. 2009, 48, 3821.

[12] Sulzer-Mossé, S.; Alexakis, A. Chem. Commun. 2007, 3123.

[13] Han, Y.; Sun, J.; Sun, Y.; Gao, H.; Yan, C. G. Chin. J. Org. Chem. 2012, 32, 1557 (in Chinese).

(韩莹, 孙晶, 孙岩, 高红, 颜朝国, 有机化学, 2012, 32, 1557.)

[14] Li, M.; Guo, W. S.; Wen, L. R.; Yang, H. Z. Chin. J. Org. Chem. 2006, 26, 1192 (in Chinese).

(李明, 郭维斯, 文丽荣, 杨华铮, 有机化学, 2006, 26, 1192.) 
[15] Wang, Y.-F.; Izawa, T.; Kobayashi, S.; Ohno, M. J. Am. Chem. Soc. 1982, 104, 6465.

[16] Neumann, J. J.; Suri, M.; Glorius, F. Angew. Chem., Int. Ed. 2010, 49, 7790.

[17] Refvik, M. D.; Schwan, A. L. J. Org. Chem. 1996, 61, 4232.

[18] Yang, Q.-Z.; Siri, O.; Braunstein, P. Chem. Commun. 2005, 2660.

[19] Lorber, C.; Vendier, L. Dalton Trans. 2013, 42, 12203.

[20] Gupta, S.; Agarwal, P. K.; Kundu, B. Tetrahedron Lett. 2010, 51, 1887.

[21] Liu, Y. Y.; Zhou, R. H.; Wan, J.-P. Synth. Commun. 2013, 43, 2475.

[22] Al-Saleh, El-Apasery, M. A.; Abdel-Aziz, R. S.; Elnagdi, M. H. J. Heterocycl. Chem. 2005, 42, 563.

[23] Zhou, Z.-Z.; Liu, F.-S.; Shen, D.-S.; Tan, C.; Luo, L.-Y. Inorg. Chem. Commun. 2011, 14, 659.

[24] Al-Saleh, B.; Abdelkhalik, M. M.; Eltoukhy, A. M.; Elnagdi, H. J. Heterocycl. Chem. 2002, 39, 1035.

[25] Reddy, G. J.; Latha, D.; Thirupathaiah, C.; Rao, K. S. Tetrahedron Lett. 2005, 46, 301.

[26] Kantevari, S.; Chary, M. V.; Vuppalapati, S. V. N. Tetrahedron 2007, 63, 13024.

[27] Kantevary, S.; Chary, M. V.; Vuppalapati, S. V. N.; Lingaiah, N. J. Heterocycl. Chem. 2008, 45, 1099.

[28] Kantevary, S.; Patpi, S. R.; Addla, D.; Putapatri, S. R.; Sridhar, B.; Yogeeswari, P.; Sriram, D. ACS Comb. Sci. 2011, 13, 427.

[29] Kantevary, S.; Addla, D.; Sridhar, B. Synthesis 2010, 3745.

[30] Kantevary, S.; Putapatri, S. R. Synlett 2010, 2251.

[31] Siddiqui, Z. N.; Ahmed, N.; Farooq, F.; Khan, K. Tetrahedron Lett. 2013, 54, 3599.

[32] Wan, J.-P.; Gan, S.-F.; Sun, G.-L.; Pan, Y.-J. J. Org. Chem. 2009,
74, 2862.

[33] Wan, J.-P.; Wang, C. P.; Pan, Y. J. Tetrahedron 2011, 67, 922.

[34] Muthusaravanan, S.; Perumal, S.; Almansour, A. I. Tetrahedron Lett. 2012, 53, 1144.

[35] Wan, J.-P.; Zhou, R. H.; Liu, Y. Y.; Cai, M. Z. RSC Adv. 2013, 3, 2477.

[36] Muthusaravanan, S.; Devi bala, B.; Perumal, S. Tetrahedron Lett. 2013, 54, 5302.

[37] Muthusaravanan, S.; Sasikumar, C.; Devi bala, B.; Perumal, S. Green Chem. 2014, 16, 1297.

[38] Petrow, V. A. J. Chem. Soc. 1946, 888.

[39] Mirza-Aghayan, M.; Langrodi, M. K.; Rahimifard, M.; Boukherroub, R. Appl. Organomet. Chem. 2009, 23, 267.

[40] Yang, J. Y.; Wang, C. Y.; Xie, X.; Li, H.-F.; Li, Y. Z. Eur. J. Org. Chem. 2010, 4189.

[41] Liu, Z. M.; Zhang, L. L.; Sun, J.; Yan, C. G. Chin. J. Chem. 2013, $31,479$.

[42] Sirijindalert, T.; Hansuthirakul, K.; Rashatasakhon, P.; Sukwattanasinitt, M.; Ajavakom, A. Tetrahedron 2010, 66, 5161.

[43] Sasada, T.; Kobayashi, F.; Moriuchi, M.; Sakai, N.; Konakahara, T. Synlett 2011, 2029.

[44] Wan, J.-P.; Liu, Y. Y. Synthesis 2010, 3943.

[45] Wan, J.-P.; Pan, Y.-J. Chem. Commun. 2009, 2768.

[46] Darwish, E. S.; Abdelhamid, I. A.; Nasra, M. A.; Abdel-Gallil, F. M.; Fleita, D. H. Helv. Chim. Acta 2010, 93, 1204.

[47] Chen, Z. G.; Wang, D.; Li, Y. N.; Wang, Y. J.; Hu, J. L.; Xia, W. Acta Chim. Sinica 2012, 70, 2236 (in Chinese).

(陈战国, 王丹, 李亚男, 王英杰, 胡均利, 夏伟, 化学学报, 2012, 70, 2236.) 